\title{
Detección y genotipificación de Pneumocystis jirovecii en muestras de pacientes mexicanos VIH positivos y negativos
}

\author{
Amanda Alcántara-Mojica, ${ }^{1}$ Laurence Delhaes, ${ }^{2}$ Fabiola Ramírez-Corona, ${ }^{3}$ Edith Sánchez-Paredes, ${ }^{1}$ \\ Érika Córdova-Martínez, ${ }^{1}$ Fernando Rosalío Morales-Villarreal, ${ }^{4}$ Sigfrido Sierra-Galván ${ }^{3}$ y \\ Francisca Hernández-Hernández ${ }^{1 *}$ \\ 'Universidad Nacional Autónoma de México, Facultad de Medicina, Departamento de Microbiología y Parasitología, Ciudad de México, México; \\ ${ }^{2}$ Universidad de Burdeos, Facultad de Medicina, Burdeos, Francia; ${ }^{3}$ Universidad Nacional Autónoma de México, Facultad de Ciencias, Ciudad de \\ México, México; ${ }^{4}$ Secretaría de Salud, Instituto Nacional de Enfermedades Respiratorias, Ciudad de México, México
}

\section{Resumen}

Introducción: Pneumocystis jirovecii es un hongo atípico detectado particularmente en pacientes VIH-positivos o con trasplante. Objetivo: Detectar y genotipificar Pneumocystis jirovecii en muestras de pacientes de dos hospitales de la ciudad de México. Método: Fueron procesadas 89 muestras respiratorias, correspondientes a 53 pacientes (30 VIH positivos y 23 VIH negativos) con sintomatología respiratoria y 11 personas sanas incluidas como control negativo. El DNA fue extraído y amplificado por PCR anidada de la región del espaciador transcrito interno, obteniendo un fragmento en cada ronda (de 693 y 550 pb). Los genotipos y su relación filogenética fueron determinados por secuenciación del fragmento de 550 pb. Resultados: Cuarenta y ocho muestras de 30 pacientes VIH-positivos provenían de un solo hospital, de las cuales 11 (36.6 \%) fueron positivas a Pneumocystis jirovecii. Ninguna fue positiva en pacientes VIH-negativos o personas sanas. Los haplotipos detectados con mayor frecuencia fueron Eg y Em. Conclusiones: La frecuencia de infección por Pneumocystis jirovecii fue alta en la población mexicana estudiada. El genotipo más frecuente fue diferente a los reportados en otros países. Es necesario encauzar este problema de salud hacia la detección temprana de esta infección.

PALABRAS CLAVE: Pneumocystis jirovecii. Neumonía. Virus de inmunodeficiencia humana. Micosis.

\section{Abstract}

Introduction: Pneumocystis jirovecii is an atypical fungus particularly detected in HIV-positive or transplant patients. Objective: To detect and genotype Pneumocystis jirovecii in patient samples from two hospitals in Mexico City. Method: Eighty-nine respiratory tract samples, corresponding to 53 patients (30 HIV-positive and 23 HIV-negative) with respiratory symptoms and to 11 healthy individuals included as negative control, were processed. DNA was extracted from the ITS region and amplified by nested polymerase chain reaction from the internal transcribed spacer, with one fragment being obtained at each round (693 and 550 bp). Genotypes and their phylogenetic relationship were determined by sequencing the 550 bp fragment. Results: Forty-eight samples from 30 HIV-positive patients were received from a single hospital, out of which 11 (36.6 \%) were positive for Pneumocystis jirovecii. No sample was positive in HIV-negative patients or healthy subjects. The most frequently detected haplotypes were $\mathrm{Eg}$ and $\mathrm{Em}$. Conclusions: The frequency of Pneumocystis jirovecii infection was high in the studied Mexican population. The most common genotype was different from those reported in other countries. It is necessary to address this health problem through early detection of this infection.

KEY WORDS: Pneumocystis jirovecii. Pneumonia. Human immunodeficiency virus. Mycosis.

Correspondencia:

*Francisca Hernández-Hernández

E-mail: frank-hh@comunidad.unam.mx
Fecha de recepción: 10-09-2018

Fecha de aceptación: 02-05-2019

DOI: 10.24875/GMM.19004675
Gac Med Mex. 2019;155:377-385

Disponible en PubMed www.gacetamedicademexico.com 


\section{Introducción}

Pneumocystis jirovecii es un hongo oportunista cuyo cultivo in vitro no se ha logrado. Causa infección pulmonar en personas inmunocomprometidas, particularmente en las infectadas con el virus de la inmunodefiencia humana $(\mathrm{VIH})$, que han desarrollado síndrome de inmunodeficiencia adquirida (SIDA), desnutridas o sometidas a trasplantantes. ${ }^{1}$ Pneumocystis jirovecii es adquirido por vía respiratoria en etapas tempranas de la vida y frecuentemente se encuentra como comensal en personas inmunocompetentes. Durante la inmunosupresión, el microorganismo se multiplica en los alveolos induciendo un proceso inflamatorio. ${ }^{2}$ La neumonía por Pneumocystis jirovecii (PCP) se manifiesta por síntomas inespecíficos, si bien la insuficiencia respiratoria es la complicación más frecuente y se asocia con alta mortalidad. ${ }^{3}$

Para detectar Pneumocystis jirovecii comúnmente se utilizan tinciones (Gomori-Grocott y Giemsa) y procedimientos moleculares. ${ }^{4,5}$ La reacción en cadena de la polimerasa (PCR) ha sido dirigida hacia la región del espaciador transcrito interno (inter transcribed spacer, ITS) del ADN ribosómico. ${ }^{2,6}$ Esta región ITS es el "código de barras" para la identificación de hongos. La comparación de secuencias de la región ITS es ampliamente usada en taxonomía y filogenia molecular porque es muy fácil de amplificar, aún a partir de cantidades pequeñas de ADN y tiene un alto grado de variación, incluso entre especies estrechamente cercanas. Este marcador existe en múltiples copias en la mayoría de las células fúngicas y es recuperable con oligonucléotidos relativamente robustos con un alto grado de confiabilidad.,

Para cada gen blanco se han reportado secuencias tipo y las diferencias detectadas en estudios similares han determinado numerosos genotipos. ${ }^{2,9,10}$ Para la región ITS1 se conocen más de 30 genotipos y para ITS2 más de $40 .^{11}$

En el mundo, la PCP representa la primera o segunda infección oportunista más común en los pacientes con infección por $\mathrm{VIH} .{ }^{12}$ En México ha sido reportada hasta en 51 y $55 \%$ de los casos de infección por VIH y el diagnóstico se ha basado en tinciones. ${ }^{13,14}$ El objetivo de esta investigación fue determinar la frecuencia de detección de Pneumocystis jirovecii y sus genotipos en muestras de pacientes infectados por VIH/SIDA o con alguna otra patología inmunosupresora, mediante PCR anidada de la región ITS seguida de secuenciación.

\section{Método}

Estudio prospectivo, transversal y prolectivo. Se solicitaron especímenes respiratorios de pacientes hospitalizados con síntomas pulmonares en el Instituto Nacional de Enfermedades Respiratorias y el Hospital Regional "General Ignacio Zaragoza" del Instituto de Seguridad y Servicios Sociales de los Trabajadores del Estado, Ciudad de México.

Se incluyeron pacientes hospitalizados, de cualquier edad, de uno u otro sexo, con síntomas respiratorios, sin tratamiento anti-Pneumocystis. No se solicitó el consentimiento informado debido a que el estudio se realizó con parte de la muestra obtenida para otros análisis. El control negativo incluyó líquido de lavado orofaríngeo de personas sanas, previa solicitud de consentimiento informado.

Para la extracción del ADN fueron consideradas las condiciones descritas por Lu et al. ${ }^{15}$ y se utilizó el kit High Pure PCR Template Preparation ${ }^{\circledR}$ (Roche, Alemania). El ADN fue almacenado a $-20{ }^{\circ} \mathrm{C}$ y después utilizado para la PCR.

\section{PCR anidada}

- Primera ronda: se utilizaron $20 \mu \mathrm{L}$ de ADN en un volumen final de $50 \mu \mathrm{L}$ que contenía $1 \mathrm{x}$ de PCR-buffer, $1.5 \mathrm{mM}$ de $\mathrm{MgCl}_{2}, 1.0 \mathrm{mM}$ de desoxirribonucleótidos trifosfato, $0.2 \mu \mathrm{M}$ de oligonucleótido 1724F (AAG TTG GTC AAA TTT GGT C), $0.2 \mu \mathrm{M}$ de oligonucleótido ITS2R (CTC GGA CGA GGA TCC TCG CC), $1.25 \mathrm{U}$ de Taq DNA polimerasa (Invitrogen, Carlsbad, California, Estados Unidos). Temperatura: $94{ }^{\circ} \mathrm{C}, 5 \mathrm{mi}-$ nutos; 35 ciclos de $94{ }^{\circ} \mathrm{C}$ por un minuto, $47{ }^{\circ} \mathrm{C}$ por un minuto y $72{ }^{\circ} \mathrm{C}$ por dos minutos; finalmente, $72{ }^{\circ} \mathrm{C}$ por cinco minutos. El tamaño del producto esperado fue de 693 pares de bases (pb). ${ }^{15}$ Como control negativo de PCR se emplearon $20 \mu \mathrm{L}$ de agua miliQ estéril y como control positivo, $3 \mu \mathrm{L}$ de DNA de una muestra notablemente positiva para Pneumocystis jirovecii por tinción de Gomori-Grocott y por PCR.

- Segunda ronda: se utilizaron $2 \mu \mathrm{L}$ del producto de amplificación de la primera ronda, en un volumen final de $50 \mu \mathrm{L}$. Los oligonucleótidos fueron ITS1F (CGT AGG TGA ACC TGC GGA AGG ATC) e ITS2R1 (GTT CAG CGG GTG ATC CTG CCT G). Las concentraciones de la mezcla de reacción fueron similares a las de la primera ronda. Temperaturas: $94{ }^{\circ} \mathrm{C}, 10$ minutos; 35 ciclos de $94^{\circ} \mathrm{C}$ 
Tabla 1. Datos generales de pacientes hospitalizados con síntomas pulmonares en el Instituto Nacional de Enfermedades

\section{Respiratorias}

\begin{tabular}{|c|c|c|c|c|c|}
\hline Número de muestra & Sexo & $\begin{array}{l}\text { Edad } \\
\text { (años) }\end{array}$ & Espécimen & Factor de riesgo & $\begin{array}{l}\text { PCR } \\
\text { Pneumocystis jirovecii }\end{array}$ \\
\hline 1 & M & - & LBA & $\mathrm{VIH} / \mathrm{SIDA}$ & Positiva \\
\hline $2 a$ & $\mathrm{~F}$ & 28 & LBA & $\mathrm{VIH}$ & - \\
\hline $2 b$ & $\mathrm{~F}$ & 28 & $\mathrm{BxP}$ & - & - \\
\hline $3 a$ & M & 25 & LBA & $\mathrm{VIH}$ & - \\
\hline $3 b$ & M & 25 & LBA & VIH, TbP & - \\
\hline $4 a$ & M & 43 & LBA & VIH/SIDA & - \\
\hline $4 b$ & M & 43 & $\mathrm{BxP}$ & VIH/SIDA & - \\
\hline 5 & $\mathrm{~F}$ & 59 & LBA & $\mathrm{VIH}$ & Positiva \\
\hline $6 a$ & M & 32 & LBA & $\mathrm{VIH}$ & Positiva \\
\hline $6 b$ & M & 32 & $\mathrm{BxP}$ & $\mathrm{VIH}$ & - \\
\hline 7 & M & 25 & LBA & VIH/SIDA, TbP & - \\
\hline $8 a$ & M & 26 & LBA & $\mathrm{VIH} / \mathrm{CM}$ & - \\
\hline $8 b$ & M & 26 & LBA & $\mathrm{VIH} / \mathrm{CM}$ & - \\
\hline $9 a$ & M & 33 & LBA & VIH & Positiva \\
\hline $9 b$ & M & 33 & $\mathrm{BxP}$ & $\mathrm{VIH}$ & - \\
\hline $10 \mathrm{a}$ & M & 26 & $\mathrm{BxP}$ & $\mathrm{VIH} / \mathrm{PCP}$ & Positiva \\
\hline $10 \mathrm{~b}$ & M & 26 & LBA & VIH/PCP & Positiva \\
\hline $11 a$ & M & 28 & LBA & VIH/PCP & Positiva \\
\hline $11 b$ & M & 28 & $\mathrm{BxP}$ & $\mathrm{VIH} / \mathrm{PCP}$ & Positiva \\
\hline $12 \mathrm{a}$ & M & 25 & $\mathrm{BxP}$ & $\mathrm{VIH} / \mathrm{SIDA}$ & Cryptococcus \\
\hline $12 \mathrm{~b}$ & M & 25 & LBA & VIH/SIDA & - \\
\hline $13 a$ & $\mathrm{~F}$ & 33 & $\mathrm{BxP}$ & VIH/PCP & - \\
\hline $13 b$ & $\mathrm{~F}$ & 33 & LBA & VIH/PCP & Positiva \\
\hline 14 & $\mathrm{~F}$ & 57 & LBA & $\mathrm{VIH}$ & - \\
\hline 15 & M & 76 & LBA & $\mathrm{VIH}$ & - \\
\hline 16 & M & 53 & LBA & Neumonía & - \\
\hline 17 & $\mathrm{~F}$ & 5 & $B \times P$ & Desconocido & - \\
\hline $18 \mathrm{a}$ & M & 44 & $B \times G$ & VIH/SK & - \\
\hline $18 b$ & M & 44 & LBA & VIH/SK & Positiva \\
\hline 19 & M & 31 & $B \times P$ & $\mathrm{VIH}$ & - \\
\hline $20 a$ & M & 28 & LBA & $\mathrm{Pb}$ VIH/PCP & - \\
\hline $20 b$ & M & 28 & $\mathrm{BxP}$ & $\mathrm{Pb}$ VIH/PCP & - \\
\hline $21 a$ & M & 44 & LBA & VIH/neumonía & Positiva \\
\hline $21 b$ & M & 44 & LBA & VIH/neumonía & - \\
\hline $22 a$ & $\mathrm{~F}$ & 31 & LBA & VIH/PCP & - \\
\hline $22 b$ & $\mathrm{~F}$ & 31 & $B \times P$ & VIH/PCP & - \\
\hline
\end{tabular}


Tabla 1. Datos generales de pacientes hospitalizados con síntomas pulmonares en el Instituto Nacioal de Enfermedades Respiratorias (Continuación)

\begin{tabular}{|c|c|c|c|c|c|}
\hline Número de muestra & Sexo & $\begin{array}{l}\text { Edad } \\
\text { (años) }\end{array}$ & Espécimen & Factor de riesgo & $\begin{array}{l}\text { PCR } \\
\text { Pneumocystis jirovecii }\end{array}$ \\
\hline $23 a$ & M & 20 & LBA & $\mathrm{Pb}$ VIH & - \\
\hline $23 b$ & M & 20 & $\mathrm{BxP}$ & $\mathrm{Pb}$ VIH & - \\
\hline $24 a$ & M & 32 & $\mathrm{BxP}$ & VIH, TbG & - \\
\hline $24 b$ & M & 32 & LBA & VIH, TbG & - \\
\hline 25 & M & 24 & LBA & VIH/PCP & Positiva \\
\hline 26 & M & 37 & LBA & VIH/PCP & Positiva \\
\hline 27 & M & 31 & $\mathrm{BxP}$ & $\mathrm{VIH} / \mathrm{Pb} \mathrm{TbP}$ & - \\
\hline 28 & $\mathrm{~F}$ & 25 & LBA & PCP & - \\
\hline 29 & M & 74 & LBA & $\mathrm{Pb} F P I$ & - \\
\hline $30 a$ & M & 42 & LBA & $\mathrm{VIH} / \mathrm{TbP}$ & - \\
\hline $30 \mathrm{~b}$ & M & 42 & $B \times G$ & $\mathrm{VIH}$ & - \\
\hline $30 c$ & M & 42 & $\mathrm{BxP}$ & $\mathrm{VIH} / \mathrm{TbP}$ & - \\
\hline
\end{tabular}

$\mathrm{M}=$ masculino, $\mathrm{F}=$ femenino, $\mathrm{LBA}=$ líquido bronquioalveolar, $\mathrm{BxP}=$ biopsia pulmonar, $\mathrm{BxG}=$ biopsia ganglionar, $\mathrm{Pb}=$ probable, $\mathrm{VIH}=$ virus de inmunodeficiencia humana,

$\mathrm{SIDA}=$ síndrome de inmunodeficiencia adquirida, $\mathrm{TbP}=$ tuberculosis pulmonar, $\mathrm{CM}=$ complejo Mycobacterium, $\mathrm{PCP}=$ neumonía por Pneumocystis, $\mathrm{TbG}=$ tuberculosis ganglionar, FPI = fibrosis pulmonar idiopática, $\mathrm{SK}=$ sarcoma de Kaposi

por un minuto, de $58{ }^{\circ} \mathrm{C}$ por un minuto, de $72{ }^{\circ} \mathrm{C}$ por dos minutos. La extensión final a $72{ }^{\circ} \mathrm{C}$ por 10 minutos. El tamaño esperado del producto fue de $550 \mathrm{pb}^{15}$

Los amplicones fueron corridos en un gel de agarosa a $1.5 \%$, teñidos con bromuro de etidio, purificados y enviados a secuenciar al Instituto de Biología de la Universidad Nacional Autónoma de México, Ciudad de México, con el método de inhibición de terminación de cadena. ${ }^{16}$ Todas las secuencias fueron realizadas por duplicado en sentidos 5'-3' y 3'-5'.

\section{Análisis de secuencias}

Las secuencias sense y antisense fueron analizadas y editadas con el programa ChromasPro y comparadas con tres bases de datos: Basic Local Alignment Search Tool del GenBank (https://www.ncbi.nlm. nih.gov/), The European Molecular Biology Laboratory (http://www.embl.de/) y Kyoto Encyclopedia of Genes and Genomes (http://www.genome.jp/kegg/).

Las secuencias fueron también analizadas con el programa MEGA versión 6.0, para construir un árbol filogenético con el método de neighbour-joining, utilizando el modelo Kimura de dos parámetros con un bootstrap de 1000 repeticiones..$^{17}$ El alineamiento múltiple se hizo utilizando ClustalW. Se construyó una matriz de distancias genéticas.

\section{Determinación de genotipos}

Se tomaron como referencia las secuencias reportadas por Lee et al., ${ }^{9}$ quien consideró 15 genotipos para la región ITS1 (A-O) y 14 genotipos para ITS2 (a-n).

\section{Resultados}

Del Instituto Nacional de Enfermedades Respiratorias se recibieron 48 muestras de 30 pacientes (23 hombres y 7 mujeres). La edad osciló de cinco a 76 años, con promedio de 35.7. La mayoría de las muestras (30) fueron líquido bronquioalveolar y tejido (18). En 26 pacientes (86.6\%), el principal factor de oportunismo fue la infección por VIH/SIDA, la mayoría de los casos confirmados (Tabla 1).

Del Hospital Regional "General Ignacio Zaragoza" se recibieron 30 muestras, correspondientes a 23 pacientes (15 hombres y ocho mujeres). La edad osciló de uno a 86 años, con promedio de 50.6. La mayoría de las muestras (25) fueron de esputo. Los pacientes presentaron diversos factores de oportunismo, con predominio de tuberculosis pulmonar (siete, $29.16 \%$ ) probable o confirmada en el momento de la recepción de la muestra (Tabla 2).

El grupo control negativo consistió en 11 muestras de líquido de lavado orofaríngeo de personas adultas, sanas, cuya edad promedio fue de 34.2 años, sin 
Tabla 2. Datos generales de pacientes con síntomas pulmonares del Hospital Regional "General Ignacio Zaragoza"

\begin{tabular}{|c|c|c|c|c|}
\hline Número de muestra & Sexo & Edad (años) & Espécimen & Factor de riesgo \\
\hline 1 & M & 50 & LBA & Desconocido \\
\hline 2 & $\mathrm{~F}$ & 64 & LBA & Desconocido \\
\hline $3 a$ & M & 38 & Esputo & Tuberculosis pulmonar \\
\hline $3 b$ & M & 38 & Esputo & Tuberculosis pulmonar \\
\hline $3 c$ & M & 38 & Esputo & Tuberculosis pulmonar \\
\hline $3 d$ & M & 38 & Esputo & Tuberculosis pulmonar \\
\hline 4 & $\mathrm{~F}$ & 52 & Esputo & Desconocido \\
\hline 5 & M & 57 & Esputo & Probable TbP \\
\hline $6 a$ & M & 37 & Esputo & Probable TbP \\
\hline $6 b$ & M & 37 & Esputo & Probable TbP \\
\hline $7 a$ & $\mathrm{~F}$ & 45 & Esputo & Tuberculosis pulmonar \\
\hline $7 b$ & $\mathrm{~F}$ & 45 & Esputo & Tuberculosis pulmonar \\
\hline 8 & M & 21 & Esputo & VIH, probable TbP \\
\hline $9 a$ & M & 63 & Esputo & Neoplasia pulmonar \\
\hline $9 b$ & M & 63 & Esputo & Hemoptisis en estudio \\
\hline 10 & M & 43 & Esputo & Tuberculosis pulmonar \\
\hline 11 & M & 31 & Esputo & $\mathrm{VIH}$ \\
\hline 12 & M & 67 & Esputo & Desconocido \\
\hline 13 & $\mathrm{~F}$ & 50 & Líquido pleural & Probable TbP \\
\hline 14 & M & 80 & Líquido pleural & Neoplasia pulmonar \\
\hline 15 & $\mathrm{~F}$ & 23 & LBA & Epilepsia \\
\hline $16 a$ & M & 57 & LBA & Neumonía \\
\hline $16 b$ & M & 57 & Esputo & Choque séptico \\
\hline 17 & $\mathrm{~F}$ & 37 & Esputo & Choque séptico \\
\hline 18 & M & 76 & Esputo & Insuficiencia respiratoria \\
\hline 19 & $\mathrm{~F}$ & 62 & Esputo & Insuficiencia respiratoria \\
\hline 20 & M & 86 & Esputo & EPOC, neumonía \\
\hline 21 & $\mathrm{~F}$ & 64 & Esputo & Neumonía \\
\hline 22 & M & 1 & Esputo & Sepsis, neumonía \\
\hline 23 & M & 61 & Esputo & Choque séptico \\
\hline
\end{tabular}

$\mathrm{M}=$ masculino, $\mathrm{F}=$ femenino, $\mathrm{LBA}=$ líquido bronquioalveolar, $\mathrm{TbP}=$ tuberculosis pulmonar, $\mathrm{VIH}=$ virus de inmunodeficiencia humana, $\mathrm{EPOC}=$ enfermedad pulmonar obstructiva crónica

antecedente de tabaquismo o síntomas respiratorios en el momento del muestreo.

\section{Detección de Pneumocystis jirovecii}

La Figura 1 muestra las estructuras parasitarias teñidas con Gomori-Grocott y el amplicon correspondiente a Pneumocystis jirovecii obtenido de líquido bronquioalveolar, el cual fue usado como control positivo de PCR. El fragmento fue purificado y secuenciado para corroborar su identidad con Pneumocystis jirovecii.

De los 30 pacientes del Instituto Nacional de Enfermedades Respiratorias, 11 fueron positivos a Pneumocystis jirovecii (Figuras 1B y 2A), todos ellos 
A

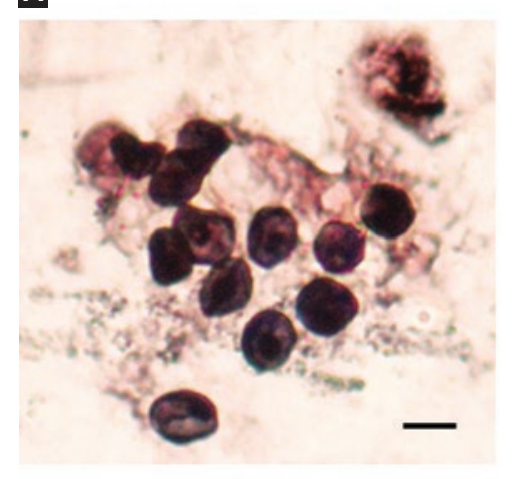

B

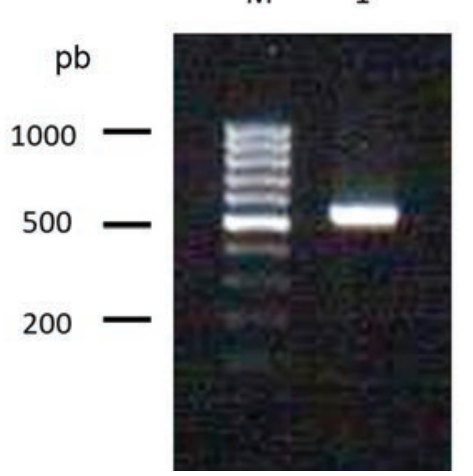

$550 \mathrm{pb}$

B

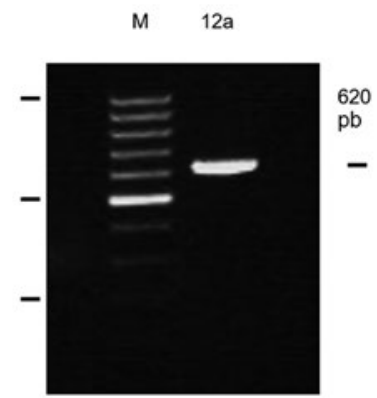

Figura 2. Amplicones de la región ITS de Pneumocystis jirovecii. A: Muestras que amplificaron un fragmento del tamaño esperado (550 pb). B: Muestra que amplificó un fragmento de mayor tamaño al esperado (620 pb). M = marcador de peso molecular de 100 pb. Los números en la parte superior indican el número de la muestra.

tenían infección por VIH como factor de oportunismo, nueve eran hombres y dos mujeres. En cinco pacientes previamente se había diagnosticado PCP por tinción de Gomori-Grocott, confirmados por PCR. Los pacientes positivos a Pneumocystis jirovecii representaron $36.6 \%$ de los casos de esta institución.

En la muestra 12a, correspondiente a un paciente del Instituto Nacional de Enfermedades Respiratorias, se obtuvo un amplicón de $620 \mathrm{pb}$, mayor al tamaño esperado (Figura 2B). Este fragmento fue secuenciado y analizado.

Todas las muestras del Hospital Regional "General Ignacio Zaragoza" y del grupo control negativo (individuos sanos) fueron negativas para Pneumocystis jirovecii.

\section{Análisis de similitud de secuencias}

La identidad con Pneumocystis jirovecii osciló entre 90 y $100 \%$. El análisis en las tres bases de datos fue similar. Todos los fragmentos amplificados correspondieron a la región ITS de Pneumocystis jirovecii. En la muestra 12a, el electroferograma fue muy confiable y el análisis comparativo indicó $87 \%$ de identidad con Cryptococcus sp.

Con los datos de la secuenciación se construyó una matriz de distancias genéticas (datos no mostrados) y con el análisis del origen de las muestras (Figura 3) se exploró su correlación. La muestra 12a se mantuvo en una rama separada de las otras. Las muestras 11a y 11b correspondieron al mismo paciente, cuyo valor de distancia genética fue 0.000; ambas agruparon en la misma rama, lo cual indica que es la misma cepa de Pneumocystis jirovecii. Las muestras 10a y 10b también pertenecían al mismo paciente, con una distancia genética de 0.152 , y se agruparon en diferentes ramas filogenéticas, lo cual sugirió que había diferencia entre las cepas de Pneumocystis jirovecii.

\section{Determinación de genotipos}

De las 14 muestras positivas a Pneumocystis jirovecii, en 11 se determinó el genotipo en ambas direcciones. En tres secuencias no fue posible determinar 


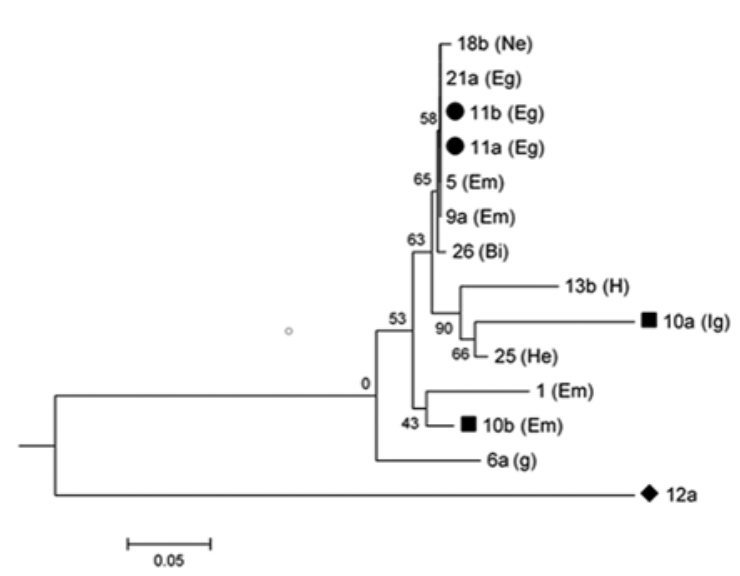

Figura 3. Árbol filogenético construido con el método de neighbour-joining. Los números corresponden a cada muestra procesada y entre paréntesis se indica su haplotipo. Las muestras 10a y 10b y 11a y $11 \mathrm{~b}$ corresponden al mismo paciente respectivamente. Cryptococcus neoformans fue identificado en la muestra 12a y fue usado como control externo.

el genotipo debido al gran número de inserciones y deleciones. En estas muestras, el procedimiento fue repetido dos veces más, desde la extracción de ADN hasta el análisis de las secuencias y los resultados fueron similares. Para ITS1 fue predominante el genotipo $\mathrm{E}$ (siete muestras) y para ITS2, el genotipo $\mathrm{g}$ (cinco muestras). Los haplotipos predominantes fueron Em (cuatro casos) y Eg (tres casos). En las muestras 10a y 10b, que correspondieron al mismo paciente, los genotipos fueron diferentes. En las muestras 11a y 11b, que también correspondieron al mismo paciente, se encontró el mismo genotipo.

\section{Discusión}

Debido a la imposibilidad para recuperar Pneumocystis jirovecii en cultivos, los procedimientos moleculares han sido particularmente útiles para detectar este organismo en muestras de pacientes en quienes se sospecha PCP. La frecuencia de detección de Pneumocystis jirovecii reportada por otros autores es variable, dependiendo de diversos factores como el tipo de muestra, la carga fúngica, el procedimiento molecular utilizado y el gen seleccionado. La PCR anidada y la PCR en tiempo real que utilizan fragmentos de ADN mitocondrial, ITS1 e ITS2 como genes blanco han mostrado altos valores de sensibilidad y especificidad. ${ }^{2,18}$

La PCR ha revelado valores de sensibilidad y especificidad para líquido bronquioalveolar que oscilan entre 84 y $100 \%$ y 58 y $100 \%$, respectivamente, ${ }^{19,20}$ por ello ha sido el espécimen más usado y confiable. Gupta et al. ${ }^{21}$ estudiaron 180 muestras de pacientes inmunosuprimidos con sospecha clínica de PCP; por PCR anidada dirigida a la región ITS encontraron $24.69 \%$ de positividad en 81 muestras de líquido bronquioalveolar y en $12 \%$ de 50 muestras de esputo. En otros países, como Brasil, por PCR anidada se ha identificado $44.8 \%$ de positividad a Pneumocystis jirovecii en pacientes VIH positivos. ${ }^{22}$ En este estudio, de 30 muestras de líquido bronquioalveolar procesados, 11 (36.6\%) fueron positivos.

Los 25 esputos que correspondieron a pacientes del Hospital Regional "General Ignacio Zaragoza" fueron negativos a Pneumocystis jirovecii. En otras investigaciones, los resultados negativos han sido atribuidos a la presencia de inhibidores de la PCR. ${ }^{23}$ Para el grupo de pacientes de ese hospital es posible que otros factores hayan influido en los resultados negativos, como el menor grado de inmunosupresión causado por la enfermedad subyacente, que fue principalmente tuberculosis pulmonar, ocasionalmente reportada en coinfección con Pneumocystis jirovecii. ${ }^{24}$ Los dos pacientes VIH positivos del Hospital Regional "General Ignacio Zaragoza" no mostraron evidencia de Pneumocystis, probablemente por una etapa moderada de inmunosupresión, como los casos de tuberculosis pulmonar.

Medrano et al. ${ }^{25}$ detectaron ADN de Pneumocystis jirovecii en 12 (20\%) de 50 muestras de líquido de lavado orofaríngeo de individuos sanos y Vargas et al. ${ }^{26}$ en $32 \%$ de 74 niños sanos. En este trabajo, la ausencia de Pneumocystis jirovecii en individuos sanos posiblemente se debió a que las personas no eran portadoras de Pneumocystis jirovecii, a que la carga fúngica era muy baja 0 a que el procedimiento de lavado orofaringeo fue inadecuado para detectar al microorganismo.

En los últimos años, las bases de datos genéticos han crecido exponencialmente y en la actualidad hay más información sobre la identidad de los microorganismos. El análisis por la herramienta de búsqueda de alineación local básica (BLASTn) de los oligonucleótidos aquí utilizados indica que amplifican para un fragmento de ADN de Pneumocystis jirovecii y Cryptococcus sp. La amplificación de un fragmento correspondiente a Cryptococcus se puede explicar porque los oligonucleótidos descritos para la región ITS son universales para la identificación molecular de especies fúngicas. ${ }^{7,8}$ Además, no es sorprendente encontrar a Cryptococcus colonizando o infectando a pacientes con infección por $\mathrm{VIH}$, ya que la levadura (principalmente Cryptococcus neoformans) es adquirida por vía respiratoria, inicialmente infecta el pulmón 
y después invade el sistema nervioso central y otros tejidos. ${ }^{27}$

La mezcla de genotipos de Pneumocystis jirovecii (como en las muestras 10a y 10b) es una condición frecuente. ${ }^{28}$ Es posible que la alta variabilidad genética y la dificultad para determinar genotipos en algunas muestras de este estudio se debiera al contacto de los pacientes con diferentes fuentes de infección y cepas. $^{29}$ Respecto a los genotipos detectados, el haplotipo Em (cuatro muestras) no ha sido reportado como predominante, diferente al haplotipo Eg (tres muestras) encontrado como el más común en otro reporte. ${ }^{30}$ Esta investigación no es suficiente para afirmar que la población mexicana sea portadora de un genotipo distinto a otras poblaciones analizadas.

Aunque en la mayoría de los estudios se utiliza esputo como espécimen, es posible que la forma de obtenerlo sea un factor decisivo para detectar al parásito fúngico. Casanova et $a l .^{13}$ encontraron Pneumocystis carinii (Pneumocystis jirovecii) en $51 \%$ de 128 pacientes después de obtener el esputo por expectoración inducida y con tinción de Giemsa.

La población infectada con VIH sigue estando en alto riesgo de desarrollar PCP debido a su bajo nivel de linfocitos CD4. ${ }^{31}$

La PCP es una patología que ocupa un lugar preponderante en la población sometida a algún factor de inmunosupresión. Se estima que en el mundo, la prevalencia anual de neumonía por Pneumocystis jirovecii es de 500000 casos. $^{32}$ Para aplicar medidas de control de las enfermedades infecciosas, en particular las infecciones oportunistas como la PCP, es necesario conocer su estado epidemiológico en cada país.

En este trabajo se identificó alta positividad de Pneumocystis jirovecii y sus genotipos en pacientes mexicanos VIH-positivos a través de la PCR anidada dirigida el gen de la región ITS. Es necesario vigilar la población mexicana en riesgo de desarrollar PCP, aplicando procedimientos moleculares para determinar con certeza la carga fúngica y, si es posible, detectar cepas resistentes a los medicamentos de uso terapéutico, para iniciar el tratamiento específico.

\section{Agradecimientos}

A la maestra en ciencias Rocío López Álvarez, del Hospital Regional "General Ignacio Zaragoza", por su apoyo en la obtención de especímenes. A la Coordinación de Investigación de la Facultad de Medicina de la Universidad Nacional Autónoma de México, por el presupuesto otorgado anualmente a la doctora Francisca Hernández Hernández.

\section{Fuente de financiamiento}

Este trabajo fue realizado con presupuesto otorgado por la Facultad de Medicina a la doctora Francisca Hernández Hernández como profesor titular "C" definitivo, de tiempo completo.

\section{Bibliografía}

1. Wang RJ, Miller RF, Huang L. Approach to fungal infections in human immunodeficiency virus-infected individuals: Pneumocystis and beyond. Clin Chest Med. 2017;38:465-477.

2. Calderón-Sandubete E, De Armas-Rodríguez Y, Capó-De Paz V. Pneumocystis jirovecii: cien años de historia. Rev Cubana Med Trop. 2011;63:97-116.

3. Vera A, Pinochet R, Villamizar G, Cancino C, Henríquez L, Cabib C. Ventilación mecánica en pacientes $\mathrm{VIH}+$ en falla respiratoria aguda. Experiencia de una Unidad de Cuidados Intensivos de reciente creación. Rev Chil Med Intensiva. 2006;21:67-72.

4. Usha M, Rakshitha HB, Avnika J, Aneesha A, Sharon R. Morphological spectrum of fungal infections: a retrospective study. Int J Med Sci Public Health. 2016;5:1673-1677.

5. López-Martínez R, Méndez-Tovar LJ, Hernández-Hernández F, Castañón-Olivares LR. Micología médica. México: Trillas; 2012.

6. Durand-Joly I, Chabé M, Soula F, Delhaes L, Camus D, Dei-Cas E. Molecular diagnosis of pneumocystis pneumonia. FEMS Immunol Med Microbiol. 2005;45:405-410.

7. Das S, Deb B. DNA barcoding of fungi using ribosomal ITS marker for genetic diversity analysis: a review. Int J Pure App Biosci. 2015;3:160-167.

8. Rodríguez-Tovar A, Xoconostle-Cázares B, Valdés M. Ecología molecular de los hongos ectomicorrízicos. Rev Fitotec Mex. 2004;27:267-278.

9. Lee CH, Helweg-Larsen J, Tang X, Jin S, Li B, Bartlett MS, et al. Update on Pneumocystis carinii f. sp. hominis typing based on nucleotide sequence variations in internal transcribed spacer regions of rRNA genes. J Clin Microbiol. 1998;36:734-741.

10. Miller RF, Lindley AR, Ambrose HE, Malin AS, Wakefield AE. Genotypes of Pneumocystis jirovecii isolates obtained in Harare, Zimbabwe, and London, United Kingdom. Antimicrob Agents Chemother. 2003;47:3979-3981.

11. Wissmann G, Morilla R, Friaza V, Calderón E, Varela JM. El ser humano como reservorio de Pneumocystis. Enferm Infecc Microbiol Clin. 2010;28:38-43.

12. Schmiedel Y, Zimmerli S. Common invasive fungal diseases: an overview of invasive candidiasis, aspergillosis, cryptococcosis, and Pneumocystis pneumonia. Swiss Med Wkly. 2016;146:w14281.

13. Casanova-Cardiel LJ, Barriga-Angulo G, Ruiz-Ordaz I, Fuentes-Allen JL. Frecuencia de Pneumocystis carinii en 128 pacientes con síndrome de inmunodeficiencia adquirida y neumonía intersticial. Diagnóstico mediante expectoración inducida. Rev Med Inst Mex Seg Soc. 1992;30:191-194.

14. García-Sancho MC, Pérez-González LE, Franco-Marina F, Reyes-Terán G. Infecciones oportunistas pulmonares en pacientes con infección por el virus de la inmunodeficiencia humana del Instituto Nacional de Enfermedades Respiratorias 1991-2001. Rev Inst Nal Enf Resp Mex. 2003;16:6-10.

15. Lu JJ, Bartlett MS, Shaw MM, Queener SF, Smith JW, Ortiz-Rivera M, et al. Typing of pneumocystis carinii strains that infect humans base on nucleotide sequence variations of internal transcribed spacers of rRNA genes. J Clin Microbiol. 1994;32:2904-2912.

16. Sanger $F$, Nicklen $S$, Coulson AR. DNA sequencing with chain-terminating inhibitors. Proc Natl Acad Sci USA. 1977;74:5463-5467.

17. Tamura K, Stecher G, Peterson D, Filipski A, Kumar S. Mega 6: molecular evolutionary genetics analysis version 6.0. Mol Biol Evol. 2013;30:2725-2729.

18. Lu JJ, Chen CH, Bartlett MS, Smith JW, Lee CH. Comparison of six different PCR methods for detection of Pneumocystis carinii. J Clin Microbiol. 1995;33:2785-2788.

19. Atzori C, Angeli E, Agostoni F, Mainini A, Micheli V, Cargnel A. Biomolecular techniques to detect Pneumocystis carinii f. sp. hominis pneumonia in patients with acquired inmunodeficiency syndrome. Int $\mathrm{J}$ Infect Dis. 1998-1999;3:76-81.

20. Álvarez-Martínez MJ, Miró JM, Valls ME, Moreno A, Rivas PV, Solé M, et al. Sensitivity and specificity of nested and real-time PCR in the detection of Pneumocystis jirovecii in clinical specimens. Diagn Microbiol Infect Dis. 2006;56:153-160. 
21. Gupta R, Mirdha BR, Guleria R, Mohan A, Kabra SK, Kumar L, et al. Use of different primer directed sequence amplification by polymerase chain reaction for identification of Pneumocystis jirovecii in clinical samples. Indian J Chest Dis Allied Sci. 2008;50:321-327.

22. Pereira RM, Müller AL, Zimerman RA, Antunes DB, Zinn VF, Friaza V, et al. High prevalence of Pneumocystis jirovecii colonization among HIV-positive patients in southern Brazil. Med Mycol. 2014;52:804-809.

23. Lundgren B, Wakefield AE. PCR for detecting Pneumocystis carinii in clinical or environmental samples. FEMS Immunol Med Microbiol. 1998;22:97-101.

24. Sheikholeslami MF, Sadraei J, Farnia P, Forozandeh-Moghadam M, Emadi-Kochak H. Co-infection of Mycobacterium tuberculosis and Pneumocystis jirovecii in the Iranian patients with human immunodeficiency virus. Jundishapur J Microbiol. 2015;8:e17254.

25. Medrano FJ, Montes-Cano MA, Conde M, De la Horra C, Respaldiza N, Gasch A, et al. Pneumocystis jirovecii in general population. Emerg Infect Dis. 2005; $11: 245-250$.

26. Vargas SL, Hughes WT, Santolaya ME, Ulloa AV, Ponce CA, Cabrera $C E$, et al. Search for primary infection by Pneumocystis carinii in a cohort of normal healthy infants. Clin Infect Dis. 2001;32:855-861.
27. Skolnik K, Huston S, Mody $\mathrm{CH}$. Cryptococcal lung infections. Clin Chest Med. 2017;38:451-464.

28. Tsolaki AG, Miller RF, Underwood AP, Banerji S, Wakefield AE. Genetic diversity at the internal transcribed spacer regions of the rRNA operon among isolates of Pneumocystis carinii from AIDS patients with recurrent pneumonia. J Infect Dis. 1996;174:141-156.

29. Sokulska M, Kicia M, Wesolowska M, Piesiak P, Kowal A, Lobo ML, et al. Genotyping of Pneumocystis jirovecii in colonized patients with various pulmonary diseases. Med Mycol. 2018;56:809-815.

30. Beser J, Botero-Kleiven S, Lebbad M, Hagblom P, Fernandez V. A limited number of ITS haplotypes defines the diversity of Pneumocystis jirovecii strains in Sweden. Infect Genet Evol. 2011;11:948-954.

31. Villasís-Keever A, Rangel-Frausto MS, Ruiz-Palacios G, Ponce de León-Rosales S. Clinical manifestations and survival trends during the first 12 years of the AIDS epidemic in Mexico. Arch Med Res. 2001;32:62-65.

32. Bongomin F, Gago S, Oladele RO, Denning DW. Global and multi-national prevalence of fungal diseases-estimate precision. J Fungi (Basel). 2017;3:57. 